\title{
Measuring Bone Density Connectivity Using Dual Energy X-Ray Absorptiometry Images
}

\author{
Li Chen ${ }^{1}$ and V. B. Surya Prasath ${ }^{2,3,4}$ \\ ${ }^{1}$ Department of Computer Science and Information Technology, University of the District of Columbia, \\ Washington, DC, USA \\ ${ }^{2}$ Computational Imaging and VisAnalysis (CIVA) Lab, Department of Computer Science, University of \\ Missouri, Columbia, MO, USA \\ ${ }^{3}$ Division of Biomedical Informatics, Cincinnati Children's Hospital Medical Center, Cincinnati, OH, USA \\ ${ }^{4}$ Department of Biomedical Informatics, College of Medicine, University of Cincinnati, OH, USA
}

\section{]jfis}

\begin{abstract}
Estimating bone density using mathematical models has direct applications in medical image processing. This paper presents a new measure for bone mineral density analysis based on the dual energy X-ray absorptiometry (DEXA) images. We are proposing an innovative procedure to calculate a scalar value that indicates the connectivity of bone mineral components from DEXA images. This method provides a totally novel measure for bone density study using DEXA scan images that can not only calculate $T$ and $Z$ values and can also determine the quality of the bone in terms of the average intensity of bone density. The new measure proposed is called the $\lambda$-measure and it provides new possibilities in terms of finding how well the bone components are connected. We can have $\lambda_{T}$ and $\lambda_{Z}$ corresponding to $T$ and $Z$ scores which augments the traditional values. Combining this method to a $\lambda$-connected maximum entropy concept, we obtain good segmentation results. The experiments results and data obtained shows that the new $\lambda$-measure should be in the range of $[0.962,0.977]$.
\end{abstract}

Keywords: Bone density, DEXA, $\lambda$-connectedness, Segmentation

Received: Aug. 25, 2017

Revised : Sep. 12, 2017

Accepted: Sep. 25, 2017

Correspondence to: Li Chen (Ichen@udc.edu)

(CThe Korean Institute of Intelligent Systems

(c) This is an Open Access article distributed under the terms of the Creative Commons Attribution Non-Commercial License (http://creativecommons.org/licenses/ by-nc/3.0// which permits unrestricted noncommercial use, distribution, and reproduction in any medium, provided the original work is properly cited.

\section{Introduction}

Bone density is an important feature to humans bone health and measuring it is an important task in medical image processing area. The mathematical models of bone density have direct consequences in possible predictions and making important diagnostics in terms of bone related treatments. Bone density is defined as the amount of bone tissue in a certain volume of bone [1]. The strength of a bone is believed to be related to and can be determined by the density of the bone structure. Moreover, bone density also measures the amount of bone mass present. It predicts fracture risks, observes the usefulness of treatments, and measures the amount of calcium in a specific region of the bone. The dual energy X-ray absorptiometry (DEXA) technique (see, Figure 11) is the most popular method used today to measure bone mineral density (BMD), which is the most important figure of bone density [2-4]. The bone density measurements allow for the diagnoses of osteoporosis and potential fracture risks at a specific site of a bone. During the DEXA test, a patient lies down on a table as shown in 
Figure 1(a). The DEXA scanner rapidly directs X-ray energy from two different sources towards the bone being examined in an alternating fashion at a set and fixed frequencies. Since two energy sources are used to measure bone density rather than the traditional X-ray or other radioisotope studies, the precision and accuracy of the measurements are greatly improved [1, 5, 7,-9].

BMD measurements usually decrease with age, and people with a BMD lower than $1.0 \mathrm{~g} / \mathrm{cm}^{2}$ need to take action in order to restore their bones. Note that, BMD does depend on the sex and age of the patient, and sometimes on the race of the patient as well. White and black females are at the greatest risk for osteoporosis or any other bone disease. Osteoporosis is known to affect 25 million people in the United States alone and half of all women over the age of 45 and past their menopause stage. It is the most common bone disease in USA today, and causes more than 1.3 million fractures including 250,000 trendy fractures, 240, 000 wrist fractures, and 500, 000 spinal fractures [10]. In addition, the National Osteoporosis Foundation recommends that BMD testing should be done on all postmenopausal women under the age of 65 who have risk factors such as a low body weight, a history of fractures, smoking, and a family history of fractures. Furthermore, all women over the age of 65 should be tested as well, whether they have risk factors or not [1, 11]. However, normal DEXA measurements only produce two individual numbers: the $T$ and $Z$ scores [12, 13]. The $T$-score is the standard deviation of the patient's BMD above or below the mean for the Young-Adult reference population. On the other hand, the $Z$-score is the standard deviation of the patient's BMD above or below the mean of the Age-Matched population. The current treatment recommends that all women start the drug therapy if they have the BMD $T$ score of less than -2 without other risk factors and for those with a $T$ score of less than -1.5 if there are other risk factors [6].

In order to understand more about human bone density, researchers have developed several methods for bone modeling and the connectivity of bone mass for the detailed analysis of the bone components, especially for the trabecular bones [14-17]. These methods include fractal analysis, finite elements, and the Monte-Carlo simulation. The finite elements method is for 3D bone modeling and is not currently directly used for DEXA images. Even though it is proven that fractal analysis is an effective measure for BMD, other methods shall be investigated intentionally to obtain a better or improved industry standard for the detailed bone density other than the classical $T$ and $Z$ scores.

In this paper, we present a new measure for BMD analysis

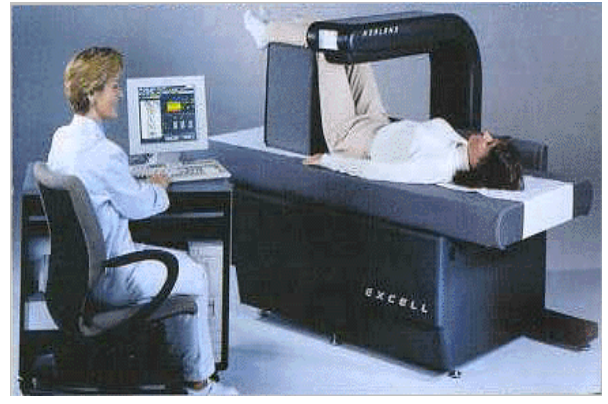

(a)
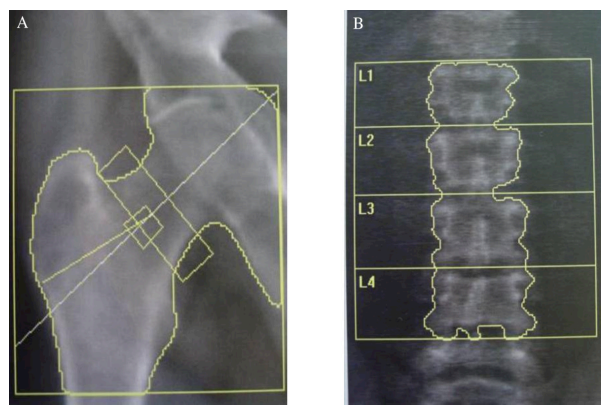

(b)

Figure 1. (a) Dual-energy X-ray absorptiometry (DEXA) procedure [5] (Dr. Caroline Lebreton, CHU Raymond Poincare, Garches, France). (b) Assessment of bone mineral density of the femoral neck (A) and the lumbar spine (B): $T$ scores of -4.2 and -4.3 were found at the hip (A) and lumbar spine (B), respectively in a 53-year-old male patient affected with Fabry disease [6].

based on DEXA images. We propose a determinative procedure to calculate a scalar value that indicates the connectivity of bone mineral components. This method provides a totally novel measure for bone density study using DEXA scan images that originally only calculates $T$ and $Z$ value that only provide the quality of the bone in terms of average intensity of bone density. The new measure proposed called $\lambda$-Measure that shows how well the bone components are connected. We could have $\lambda_{T}$ and $\lambda_{Z}$ corresponding to $T$ and $Z$ scores. Combining with this method to $\lambda$-connected maximum entropy, we can see the similar result is obtained. The data experiments showed that $\lambda$-measure should be in the range $[0.962,0.977]$. This opens up new research directions in terms of bone mineral connectivity as well as density analysis from DEXA images. A preliminary research can be found in [18].

\section{Background}

Bone density is the amount of bone tissue in a certain volume of bone $\left(\mathrm{g} / \mathrm{cm}^{3}\right)$. (See Figure 2[1]) It is often very difficult to 


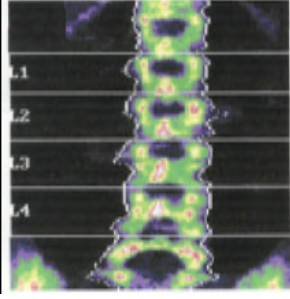

(a) The four lumbar

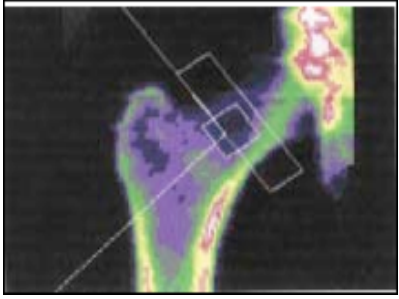

(b) The hipbone

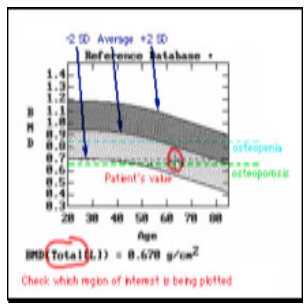

(c) The DEXA report

Figure 2. Typical DEXA scan images and report with annotations [13].

establish a measure without damaging the bone; therefore, the $\operatorname{BMD}\left(\mathrm{g} / \mathrm{cm}^{2}\right)$ is used instead [10, 13]. The strength of a bone can be determined based on BMD. In addition, BMD measures the amount of bone mass, predicts fracture risks, observes the usefulness of treatment, and measures the amount of calcium in a specific region of the bone. BMD can be measured using DEXA, the most accurate and less expensive technology available today. The normal average BMD is about $3.88 \mathrm{~g} / \mathrm{cm}^{2}$ in males and $2.90 \mathrm{~g} / \mathrm{cm}^{2}$ in females. The range of BMD in the forearm is from 700 to $800 \mathrm{~g} / \mathrm{cm}^{2}$ and in the spine region is 1,000 to $1,200 \mathrm{~g} / \mathrm{cm}^{2}$. The density of hard bone is a constant number of $1,900 \mathrm{~kg} / \mathrm{m}^{3}$ [10]. Considerable amount of research are conducted in this area [10, 15, 19, 24].

Three main companies that manufacture DEXA detector densitometers are: Hologic, Norlan, and Lunar. Usually, the detector scans two or more areas, the hip, spine and/or total body. Figure 2 (a) shows the division of the four lumbar and Figure 2 (b) shows a picture of a hipbone that was scanned by a Lunar-machine, and Figure 2 (c) shows a report made by [13].

After the bone image has been scanned onto the computer screen, an algorithmic program in the QDR software automatically computes the results based on the bone scan using the following formula: The $T$-score is the standard deviation (SD) of the patient's BMD above or below the mean for the YoungAdult normal reference population. The $Z$-score is the SD of the patient's BMD above or below the mean of the Age-Matched

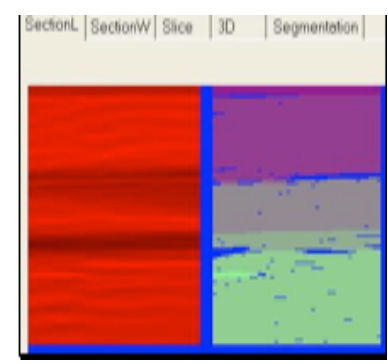

(a)

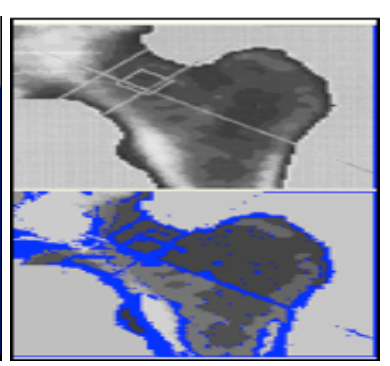

(b)
Figure 3 . The $\lambda$-connectedness based image segmentations provides better region separation using a discrete connected measure as illustrated by (a) seismic image with three distinct regions separated by interfacial boundaries and (b) DEXA image which is also segmented accurately with different gray regions representing the density levels along with boundaries given in blue color. For color referred in the figure, the reader is referred to the PDF version of the article.

population:

\section{$T$ Score}

$$
=\frac{(\text { BMD of patient }- \text { Average BMD of Young-Adult })}{\text { SD of Young-Adult }},
$$

$Z$ Score

$$
=\frac{(\text { BMD of patient }- \text { Average BMD of Age-Matched })}{\text { SD of Age-Matched }} \text {. }
$$

\section{Connected Component Segmentation}

Image segmentation is a task which requires a scheme to partition an image into connected components with similar characteristics [25]. Each component is a targeted object. There are many kinds of segmentation, e.g. measurement of space clustering, region growing, split-and-merge segmentation, edge detection, active contours, etc. Different types of data need different segmentation methods. For gray-scale images such as velocity profiles, we can use region growing or edge detection. For texture images such as waveform profiles or seismic sections, a rule-based segmentation scheme may be needed.

A digital image $F$ is a mapping from a grid-space $\Sigma$ to the real set $\mathbb{R}$ (or $\mathbb{R}^{n}$ in general). Two points $p, q$ in $\Sigma$ are adjacent if the distance between these two points is equal to 1 . Two points $p, q$ in $S \subset \Sigma$ are connected if there is a path which consists of adjacent points that links $p$ and $q$. The set $S$ is connected if each pair of points in $S$ is connected. We say $S$ is uniform if $F$ on $S$ (a sub-image) has properties of uniformity. For instance, a popular uniformity measure is defined in terms 
of the maximum difference between any pixel value and the mean value of $S$. Segmentation can be defined as a partition of an image into connected subsets, each of which is uniform, but no union of adjacent subsets is uniform. The formal definition of segmentation is then: In a digital image $F$, if there exists a non-empty segmentation $F_{1}, F_{2}, \ldots, F_{m}$, satisfying

1) $F_{i} \cap F_{j}=\emptyset$, if $i=1, \ldots, m$, and $j=1, . ., m, i \neq j$,

2) $\cap_{i=1, \ldots, m} F_{i}=F$,

3) Each $F_{i}$ is connected,

4) Each $F_{i}$ is uniform,

5) If $F_{i}$ and $F_{j}$ are adjacent, then $F_{i} \cup F_{j}$ is not uniform.

Then, $\left\{F_{1}, F_{2}, \ldots, F_{m}\right\}$ is called a segmentation of the given image $F$ and each $F_{i}$ represent a connected region.

We next briefly describe a discrete connected component based segmentation known as $\lambda$-connected segmentation. The $\lambda$-connected segmentation uses a graph-theoretic method to find a connected component in an image when only small changes appear in adjacent pixels [26-33]. It is partially an inversion of gradually varied fitting [34]. The technique is defined on a graph as $G=(V, E)$ with an associated potential function $\alpha_{\epsilon}$. A measure $C(x, y)$ is defined in $\lambda$-connectedness on two vertices $x, y \in G$. For $\lambda \in[0,1], x$ and $y$ are said to be $\lambda$ connected if $C(x, y) \geq \lambda$. Figure 3 a) shows an application on seismic image segmentation with $\lambda=0.98$. The left frame is the original image, and the right frame shows the five layers of segmented image. Each layer represents a connected component present in the image. This program can segment bone density images (See Figure 3 b), the original picture shown at top is from a DEXA scan image). Note the similarities in both the images, the objective is to segment distinct regions according to their grayscale value distribution. We will explain how the bone image analysis can be done using the generic $\lambda$ connectedness in the following section in more detail. We recall the definitions and results associated with $\lambda$-connectedness and refer to [35, Chapter 12] for more details.

Suppose that $F$ is a digital image. Then, $F$ is a mapping: $F: \Sigma_{2} \rightarrow \mathbb{R}$, where $\Sigma_{2}$ is a $2 D$ array and $\mathbb{R}$ is a set of real numbers. Thus, a pixel is a point in $\Sigma_{2} \times \mathbb{R}$. Let $d(x, y)$ be any standard distance function, in this work we use the Euclidean distance.

Definition 1. Let $\epsilon$ be a small real number. If $p=(x, F(x))$ and $q=(y, F(y))$ satisfy $d(x, y) \leq \epsilon$, then $p$ and $q$ are called $\epsilon$-neighbors.
Definition 2. If a function $\alpha_{\epsilon}: P \times P \rightarrow[0,1]$, and $p=$ $(x, F(x))$ and $q=(y, F(y))$ satisfy

1) $\alpha_{\epsilon}(p, p)=1$,

2) $\alpha_{\epsilon}(p, q)=\alpha_{\epsilon}(q, p)$,

3) $\alpha_{\epsilon}(p, q)=0$, if $d(x, y)>\epsilon$,

4) $\alpha_{\epsilon}(p, q) \geq \alpha_{\epsilon}\left(p, q^{\prime}\right)$, if $d(f(x), f(y)) \leq d\left(f(x), f\left(y^{\prime}\right)\right)$,

then $\alpha_{\epsilon}$ is called the neighbor-connectivity with respect to $\epsilon$.

A finite sequence $\Omega\left(p\left(x_{1}\right), p\left(x_{n}\right)\right)=\left\{p\left(x_{1}\right), p\left(x_{2}\right), \ldots, p\left(x_{n}\right)\right\}$ is called an $\epsilon$-path, if $d\left(x_{i}, y_{j}\right) \leq \epsilon, j=i+1$ and $x_{i} \neq x_{j}$, $i \neq j$ except that $x_{1}=x_{n}$. The neighbor-connectivity of an $\epsilon$-path $\Omega\left(p\left(x_{1}\right), p\left(x_{n}\right)\right)$ is defined as

$$
\begin{aligned}
& \alpha_{\epsilon}\left(\Omega\left(p(x), p\left(x_{n}\right)\right)\right) \\
& =\min \left\{\alpha_{\epsilon}\left(p\left(x_{i}\right), p\left(x_{i}+1\right)\right): i=1, \ldots, n-1\right\},
\end{aligned}
$$

or

$$
\begin{aligned}
& \alpha_{\epsilon}\left(\Omega\left(p(x), p\left(x_{n}\right)\right)\right. \\
& =\Pi \alpha_{\epsilon}\left(p\left(x_{i}\right), p\left(x_{i}+1\right)\right), i=1, \ldots, n-1 .
\end{aligned}
$$

Finally,

$$
\begin{aligned}
& C(p(x), p(y)) \\
& =\max \left\{\alpha_{\epsilon}(\Omega(p(x), p(y)): \Omega(p(x), p(y)) \text { is an } \epsilon-p a t h\}\right.
\end{aligned}
$$

is called the connectivity of $p(x)$ and $p(y)$ with respect to the $\epsilon$-paths.

Definition 3. $p(x)$ and $p(y)$ are called $\lambda$-connected if

$$
C(p(x), p(y)) \geq \lambda, \quad \lambda \in[0,1]
$$

The following theorem shows the characteristics of the $\lambda$ connectedness according the neighborhood connectivity rule used.

Theorem 1. The $\lambda$-connectedness is an equivalence relation if $(3)$ is used, i.e., it can be used for classification. The $\lambda$ connectedness is a similarity relation if (4) is used.

\section{Maximum Entropy Criterion for $\lambda$ Estima- tion}

According to a study [36], we use the maximum entropy method to determine the $\lambda$ value. It is $\lambda$-connected maximum entropy. 
The maximum entropy method was first proposed by Kapur et al. [37]. It is based on the maximization of inner entropy in both the foreground and background. The purpose of finding the best threshold is to make both objects in the foreground and background, respectively, as smooth as possible. Such an idea can be used for $\lambda$-connected segmentation. However, the total inner entropy for the image is to calculate the entropy for each segment ( $\lambda$-connected component), not for the thresholding clipped foreground/background. This is because in $\lambda$-connected segmentation there is no specific background. Each $\lambda$-connected segment can be viewed as foreground, and the rest may be viewed as the background. It is different from the original maximum entropy where the range of pixel values determines the inclusion of pixels. Therefore, we need to summarize all inner entropies in all segments.

$H(\lambda)=\sum$ (inner entropy of each $\lambda-$ connect. comp.).

We will select the $\lambda$ such that $H(\lambda)$ will be maximized. We call this $\lambda$-value the maximum entropy connectedness. This unique value is a new measure for images. Since the maximum entropy means the minimum amount of information or minimum variation, we want the minimum change inside each segment. This matches the philosophy of the original maximum entropy method. In other words, the $\lambda$-connected maximum entropy has a better meaning in some applications. We use the $\lambda_{e}$ such that

$$
H\left(\lambda_{e}\right)=\max \{H(x): x \in[0,1]\} .
$$

Assume there are $m$ number of $\lambda$-components, define inner entropy of each $\lambda$-component $S_{i}$ :

$$
H_{i}(\lambda)=\sum_{k=0}^{255}(- \text { Histogram }[k] / n)(\log (\operatorname{Histogram}[k] / n)),
$$

where $n$ is the number of points in the component $S_{i}$. Histogram $[k]$ is the number of pixels whose values are $k$ in the segment. Thus,

$$
H(\lambda)=\sum_{i=1}^{m} H_{i}(\lambda)
$$

Figure 4 shows the result for the DEXA image shown in Figure 3 b), using the maximum entropy connectedness presented in this section, we got $\lambda_{e}=0.95$. Finally, Figure 5 shows an example of a four part lumbar data and its corresponding

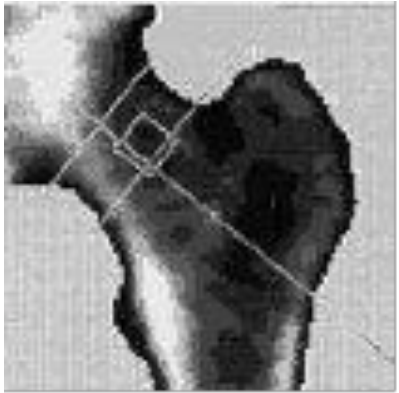

Figure 4. Maximum entropy criteria, $\lambda_{e}=0.95$ based result of $\lambda$-connected segmentation.

$\lambda$-connected segmentation results for various $\lambda$ values. The original image shows a good strength bone. Figure 5 b),$\lambda=0.98$, contains a some separated segmentation in each lumbar especially in the leftmost lumbar. It means $\lambda=0.98$ is too large Figure $5(\mathrm{c}), \lambda=0.97$, and Figure $5(\mathrm{~d}), \lambda=0.96$, showed the good connectivity. Figure 5 (e), $\lambda=0.95$, and Figure 5 (f), $\lambda=0.93$, have connected more data points that may be noisy pixels. This example also proved that the previous result of $[0.962,0.977]$ range for the $\lambda$-measure is reasonable. A large data set for running the methods described above are necessary for obtaining a more accurate estimation and more research shall be done in the future.

\section{A New Measure for Bone Connectivity}

To determine the value of $\lambda$ in segmentation is a critical issue. How to determine the value of $\lambda$ that will yield a clear segmentation? The question is even though we can find such a $\lambda$ by test a numbers of different value in segmentation. The value is not unique. We need to find a definitive procedure to determine the value of $\lambda$.

The selection of $\lambda$ depends on how many segments we want to separate. In the following section, we will discuss the maximum-connectivity spanning tree method, which can be used to find the value of $\lambda$. Since the process of finding the maximum-connectivity spanning tree is time consuming, a histogram or an experimental test may be initiated to solve the problem. A fast algorithm to find the value of $\lambda$ is the binary search. It is used to test the value of $\lambda$. First, test for $\lambda=0.5$. If $\lambda$ is too big then $\lambda=0.25$ is tested. If $\lambda$ is too small then $\lambda=0.75$ is tested and so on. Similarly, for $\lambda$-connected fitting, the same method may also be used. The speed of the binary search method compared to the minimal spanning tree method for different sized bone images will also be studied. Finding 


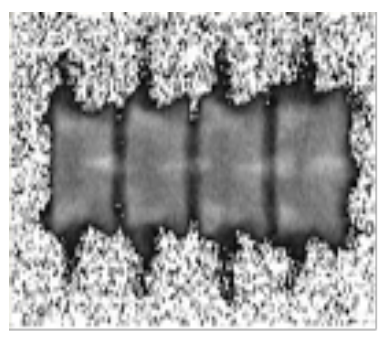

(a) Original

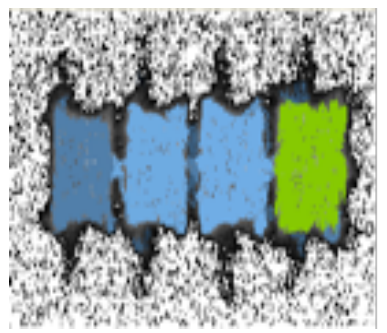

(c) $\lambda=0.97$

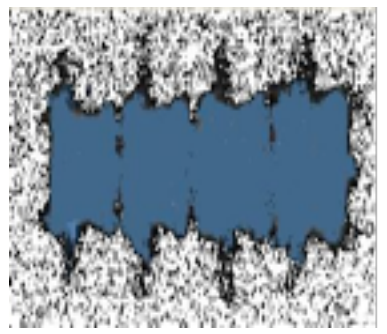

(e) $\lambda=0.95$

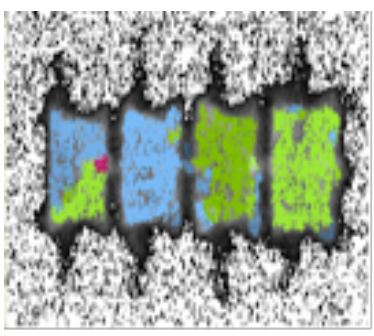

(b) $\lambda=0.98$

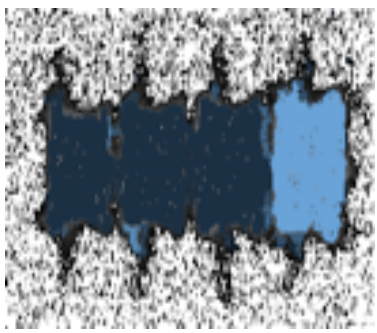

(d) $\lambda=0.96$

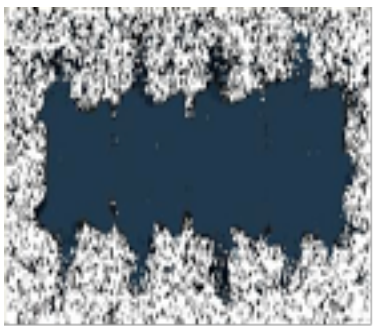

(f) $\lambda=0.93$
Figure 5. Four part lumbar data and its $\lambda$-connected component segmentations show in different color codings. The segmentation results in (c), and (d) show good connectivity, and represent good results whereas the results in (b) is fragmented, and (e)-(f) include noisy pixels in the segments. For color referred in the figure, the reader is referred to the PDF version of the article.

the corrected $\lambda$ value for different bones or for a different age group of patients is more important than finding a value for $\lambda$ in terms of good segmentation. The value that can lead to segment a DEXA-image in a good understandable component may indicate the quality of the bone, just like the fractal dimensions. An appropriate $\lambda$ value means to collect each bone component correctly. A large amount of bones from the young adults or a different age group may be needed to determine the best $\lambda$ value. The best $\lambda$ value means that it produces the satisfied bone components and the $\lambda$ value is not too big or too small. The relationship between the $\lambda$ value and the value of fractal dimensions is another important issue. If there is a clear relationship, the result will be very impressive at the science level. It could lead to new developments in human bone density analysis research.

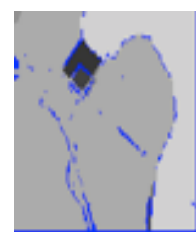

(a) $\lambda=0.93$

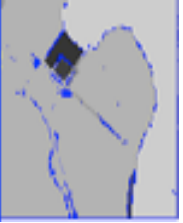

(b) $\lambda=0.94$

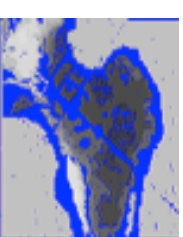

(e) $\lambda=0.98$

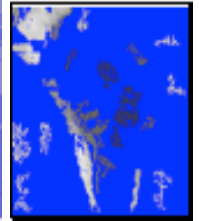

(c) $\lambda=0.99$

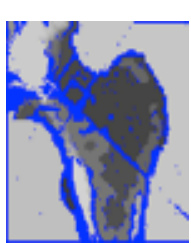

(f) $\lambda=0.97$

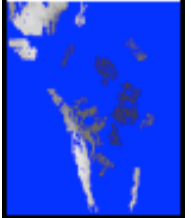

(d) $\lambda=1$
Figure 6. Different $\lambda$ values and their corresponding segmentation results. Note that the blue color indicates the border and background of the segments. For color referred in the figure, the reader is referred to the PDF version of the article.

When $\lambda$ is small, the segmentation is rough. When $\lambda$ is big, the segmentation is fine. We define

$$
\begin{aligned}
& \lambda_{\min }=\{\text { the largest value that segments } \\
& \text { the bone into only one component }\}, \\
& \lambda_{\max }=\{\text { the smallest value that segments } \\
& \text { the bone is as the same as when } \lambda=1\} .
\end{aligned}
$$

The measure $\lambda_{m}=0.5\left(\lambda_{\min }+\lambda_{\max }\right)$. Or we can choose Golden-Cut-Ratio $\lambda_{.618}=\left(.618 \lambda_{\min }+.382 \lambda_{\max }\right)$ and $\lambda^{.618}=$ $\left(.618 \lambda_{\max }+.382 \lambda_{\min }\right)$. With this value if it produces more segments that means the bone connectivity is not good. If it produces less segments, it means that the bone connectivity is good.

$$
\begin{aligned}
& \lambda_{T} \text { Score } \\
& =\frac{\left(\lambda_{m} \text { of patient }- \text { Average } \lambda_{m} \text { of Young-Adult }\right)}{\text { SD of Young-Adult }}, \\
& \lambda_{Z} \text { Score } \\
& =\frac{\left(\lambda_{m} \text { of patient }- \text { Average } \lambda_{m} \text { of Age-Matched }\right)}{\text { SD of Age-Matched }} .
\end{aligned}
$$

Compared to the original measures given in Eqs. (1) and (2), we see that our new measure is based on the $\lambda$-connectedness thereby discerned directly from the DEXA images. The new measures are therefore robust as it depends on the data itself and can be computed automatically. Further, the the SD of YoungAdult and SD of Age-Matched are kept the same between the original scores and the new measure considered here. For ex- 


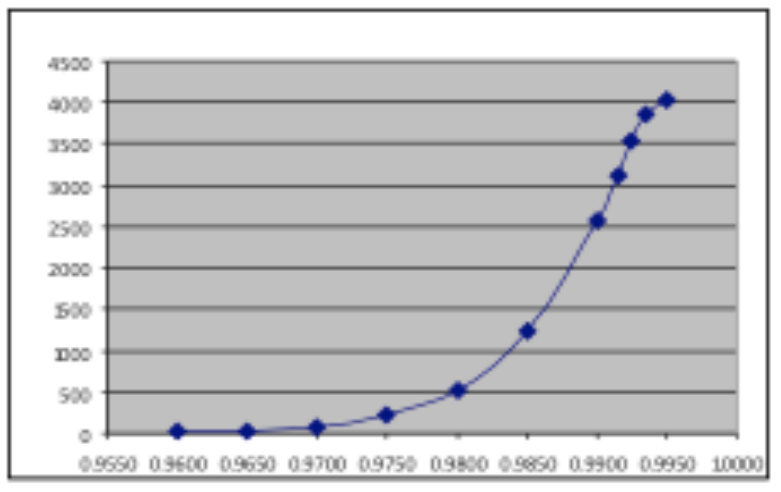

Figure 7. Different $\lambda \in[0,1]$ values and the number of segments based on Table 1 The index of $x$ and $y$ axis represent the $\lambda$ value and the corresponding number of segments obtained respectively. Note that we restricted our range of the $\lambda$ between 0.9600 to 0.9950 , and the number of segmentations above 22 .

ample, in Figure 6 we check the $\lambda$-measure with the example showed in Figure 3 b). In this image, we do not display any segment that is smaller than 30 pixels. So, $\lambda_{\min }=0.940, \lambda_{\max }=$ 1 , and $\lambda_{m}=0.970 . \lambda_{.618}=\left(.618 \lambda_{\min }+.382 \lambda_{\max }\right)=0.962$ and $\lambda^{.618}=\left(.618 \lambda_{\max }+.382 \lambda_{\min }\right)=0.977$. The measure of connectivity should be place in the range of $[0.962,0.977]$. The Table 1 lists the calculation results based on different $\lambda$-values and Figure 7 shows the corresponding graph. Based on this curve, we suggest a modeling function for the distribution.

$$
P(\lambda)=p_{0} d\left(\lambda-\lambda_{\min }\right) /\left(\delta_{\lambda}\right)+c
$$

where $\lambda_{\min } \leq \lambda \leq \lambda_{\max }$ and $d$ is the dimension of the image. Again, $\lambda_{\min }$ is the maximum $\lambda$-value making the image to be only one segment, and $\lambda_{\max }$ is the minimum $\lambda$-value making the most of segments (It also can count the small percentage cut at the each end).

Remark 1. Note that the $\lambda$-connected segmentation is to segment an image if a value of $\lambda$ is provided. $\lambda$-measure is to find a $\lambda$ value that is the best to describe the connectivity or connectedness of an image. $\lambda$-connected segmentation is basically a dual segmentation technique comparing to the (multiple-) threshold segmentation method that translates a gray-scale image into a binary image based on a clip-level.

One can think about the thresholding as a vertical processing technique, but $\lambda$-connected search is a horizontal search. These two methods share the following properties: (1) they are simple to implement, and (2) The segmentation results generate equivalence relations. They can be cooperated by (a) using $\lambda$ -
Table 1. Partitioned segments according to the $\lambda$ values

\begin{tabular}{cccc}
\hline$\lambda$ & $P$ & $\sqrt{P}$ & $\log P$ \\
\hline 0.9600 & 22 & 4.690416 & 1.342423 \\
0.9650 & 27 & 5.196152 & 1.431364 \\
0.9700 & 64 & 8 & 1.80618 \\
0.9750 & 215 & 14.66288 & 2.332438 \\
0.9800 & 526 & 22.93469 & 2.720986 \\
0.9850 & 1237 & 35.17101 & 3.09237 \\
0.9900 & 2565 & 50.64583 & 3.409087 \\
0.9915 & 3123 & 55.88381 & 3.494572 \\
0.9925 & 3547 & 59.5567 & 3.549861 \\
0.9935 & 3847 & 62.02419 & 3.585122 \\
0.9950 & 4020 & 63.40347 & 3.604226 \\
\hline
\end{tabular}

Table 2. Simple illustration of $\lambda$-connectedness and thresholding (example of the $\lambda$-connected component of $P$ has two $\lambda$-connected components after thresholding)

\begin{tabular}{ccccccccc}
\hline \multicolumn{3}{c}{ Orignal $P$} & \multicolumn{3}{c}{ Variation 1} & \multicolumn{3}{c}{$T_{1,6}$} \\
\hline 5 & 6 & 5 & 5 & 6 & 5 & 5 & & 5 \\
4 & 0 & 1 & 4 & & 1 & 4 & & 1 \\
3 & 2 & 1 & 3 & 2 & 1 & 3 & 2 & 1 \\
\hline
\end{tabular}

connected segmentation first (to generate major segments) then multiple-thresholding (to generate detailed segments), or (b) using multiple-thresholding first (to generate major segments) then $\lambda$-connected segmentation (to generate detailed segments). If we use the same $\lambda$-value and thresholds, then we would guess that we will get the same detailed segments. This statement is not true since we have the following counter-example:

Mathematically, let $T_{a, b}(P)$ be the threshold result for all pixel in image $P$ that is greater than or equal to $a$ and smaller than $b$, and let $S_{\lambda}(P)$ be the results of $\lambda$-connected segmentation respect to value $\lambda$. Thus, apply $\lambda$-connected segmentation then use thresholding is denoted by $T_{a, b}\left(S_{\lambda}(P)\right)$. Two pixels in $T_{a, b}(P)$ is $\lambda$-connected then they must be $\lambda$-connected in $P$. Therefore, $S_{\lambda}\left(T_{a, b}(P)\right)$ is the refinement of $T_{a, b}\left(S_{\lambda}(P)\right)$, see Table 2 for illustration.

Theorem 2. $S_{\lambda}\left(T_{a, b}(P)\right) \leq_{\text {ref }} T_{a, b}\left(S_{\lambda}(P)\right)$.

Remark 2. Applying $\lambda$-connectedness based segmentation result in finding the appropriate thresholds quickly. Whereas to apply thresholding first will help in stopping the extreme region linking in the segmentation. 


\section{Conclusion}

We considered a new measure for bone density based on $\lambda$ connectedness in DEXA images. Our proposed method can not only calculate BMD scores for the patients, but also determines the quality of the bon in terms of the average intensities. Our main aim here was to derive a new measure for BMD analysis based on the $\lambda$-connectedness analysis and comparisons with the original $T$ - and $Z$-scores created from the DEXA exams is required on a larger dataset for understanding the upgraded guidance obtained by our automatic BMD calculations. For a general image, the best description of the image connectivity, may still be a $\lambda$-measure. However, the $\lambda$ range might only be determined in a small range, how to find this range? Can we use information theory or statistical methods to this problem? Note that it is related to the research on the image pyramid and scale space based segmentation schemes [38--40]. Some new investigation on bone density research can be found in [41]. These research directions are worthwhile to pursue in the future, along with the new developments on graph-based segmentation are still active, and it can be found in [42].

\section{Conflict of Interest}

No potential conflict of interest relevant to this article was reported.

\section{Acknowledgement}

Many thanks to Drs. Richard Squier and Chin-Shoou Lin for providing helps in this research. The testing image in Figure 5(a) was from NIH.

\section{References}

[1] N. V. Chawla, "A preliminary paper on dual-energy X-ray absorptiometry (DEXA)," Independent Research Tutorial, Georgetown University, 2003.

[2] R. Lorente-Ramos, J. Azpeitia Arman, A. Munoz Hernandez, J. M. Garcia Gomez, P. Diez-Martinez, and M. Grande-Barez, "Dual-energy X-ray absorptiometry in the diagnosis of osteoporosis: a practical guide," American Journal of Roentgenology, vol. 196, no. 4, pp. 897904, 2011. https://doi.org/10.2214/ajr.10.5416

[3] R. M. Lorente Ramos, J. Azpeitia Arman, N. Arevalo Galeano, A. Munoz Hernandez, J. M. Garcia Gomez, and
J. Gredilla Molinero, “Absorciometria con rayos X de doble energia. Fundamentos, metodologia y aplicaciones clnicas," Radiologia, vol. 54, no. 5, pp. 410-423, 2012. https://doi.org/10.1016/j.rx.2011.09.023

[4] C. C. Gluer, "30 years of DXA technology innovations," Bone, vol. 104, pp. 7-12, 2017. https://doi.org/10.1016/j. bone.2017.05.020

[5] "What is DEXA Scanning," Available http://www gorhams.dk/html/what_is_dexa_scanning.html

[6] D. P. Germain, "Fabry disease," Orphanet Journal of Rare Diseases, vol. 5, article no. 30, 2010. https://doi.org/10. 1186/1750-1172-5-30

[7] R. S. Lu, M. Taylor, and M. J. Bottema, “Texture analysis improves the estimate of bone fracture risk from DXA images," Osteoarthritis and Cartilage, vol. 24, pp. S319S320, 2016. https://doi.org/10.1016/j.joca.2016.01. 571

[8] R. B. Staron, R. Greenspan, T. T. Millar, J. P. Bilezikian, E. Shane, and N. Haramati, "Computerized bone densitometric analysis: operator-dependent errors," Radiology, vol. 211, no. 2, pp. 467470, 1999. https://doi.org/10.1148/ radiology.211.2.r99ma55467

[9] A. P. Spence, Basic Human Anatomy. Redwood City, CA: Benjamin/Cummings, 1990.

[10] A. Yarusskaya and K. Donina Density of bone,' 2002; Available https://hypertextbook.com/facts/2002/ AnnaYarusskaya.shtml

[11] UpToDate, "Results for bone density testing beyond the basics," Available http://www.uptodate.com/contents/ bone-density-testingbeyond-the-basics

[12] Bone density," 2013; Available http://courses.washington edu/bonephys/opbmd.html

[13] DEXA reports," 2002; Available http://courses washington.edu/bonephys/opDEXA1.html

[14] M. L. Richardson and T. Gillespy, "Fractal analysis of trabecular bone," Available http://www.fractal.org/LifeScience-Technology/Publications/Fractal-Analysis-ofTrabecular-Bone.htm 
[15] R. Ruimerman, B. van Rietbergen, P. A. J. Hilbers, and R. Huiskes, "A 3-dimensional computer model to simulate trabecular bone metabolism," Biorheology, vol. 40, no. $1-3$, pp. 315320, 2003.

[16] J. S. Thomsen, J. Barlach, and L. Mosekilde, “Determination of connectivity density in human iliac crest bone biopsies assessed by a computerized method," Bone, vol. 18, no. 5, pp. 459465, 1996. https://doi.org/10.1016/87563282(96)00048-8

[17] G. J. Michael, L. H. Sim, and T. van Doorn, "A Monte Carlo model for bone mineral measurement using dual energy X-ray absorptiometry," Australasian Physical \& Engineering Sciences in Medicine, vol. 20, no. 2, pp. 8491, 1997.

[18] L. Chen, "-measure for bone density connectivity," in Proceedings of IEEE International Symposium on Industrial Electronics, Montreal, Canada, 2006, pp. 489494. https://doi.org/10.1109/ISIE.2006.295644

[19] O. C. Yeh and T. M. Keaveny, "Biomechanical effects three-dimensional finite element study,' Bone, vol. 25, no. 2, pp. 223228, 1999. https://doi.org/10.1016/s87563282(99)00092-7

[20] E. Verhulp, B. van Rietbergen, and R. Huiskes, "A threedimensional digital image correlation technique for strain measurements in microstructures," Journal of Biomechanics, vol. 37, no. 9, pp. 13131320, 2004. https://doi.org/10. 1016/j.jbiomech.2003.12.036

[21] J. Samarabandu, R. Acharya, E. Hausmann, and K. Allen, "Analysis of bone X-rays using morphological fractals," IEEE Transactions on Medical Imaging, vol. 12, no. 3, pp. 466470, 1993. https://doi.org/10.1109/42.241873

[22] S. Majumdar, J. Lin, T. Link, J. Millard, P. Augat, X. Ouyang, et al., "Fractal analysis of radiographs: assessment of trabecular bone structure and prediction of elastic modulus and strength," Medical Physics, vol. 26, no. 7, pp. 13301340, 1999. https://doi.org/10.1118/1.598628

[23] G. J. Michael and C. J. Henderson, "Monte Carlo modeling of an extended DXA technique," Physics in Medicine and Biology, vol. 43, no. 9, pp. 25832596, 1998. https: //doi.org/10.1088/0031-9155/43/9/011
[24] J. H. Kinney and A. J. Ladd, "The relationship between three-dimensional connectivity and the elastic properties of trabecular bone," Journal of Bone and Mineral Research, vol. 13, no. 5, pp. 839845, 1998. https://doi.org/ 10.1359/jbmr.1998.13.5.839

[25] R. C. Gonzalez and R. Wood, Digital Image Processing. Reading, MA: Addison-Wesley, 1993.

[26] L. Chen, O. Adjei, and D. H. Cooley, " $\lambda$-Connectedness: method and application," in Proceedings of IEEE Conference on System, Man, and Cybernetics, Nashville, TN, 2000, pp. 11571562. https://doi.org/10.1109/icsmc.2000 886243

[27] L. Chen, "Gradually varied surface and its optimal uniform approximation," in Image and Video Processing II, vol. 2182, pp. 300307, 1994. https://doi.org/10.1117/12. 171078

[28] L. Chen, "The -connected segmentation and the optimal algorithm for split-and-merge segmentation," Chinese Journal of Computers, vol. 14, no. 5, pp. 321331, 1991.

[29] L. Chen, " $\lambda$-connected approximations for rough sets," in Rough Sets and Current Trends in Computing. Heidelberg: Springer, 2002, pp. 572577. https://doi.org/10.1007/3540-45813-1_76

[30] L. Chen, F. T. Berkey, and S. A. Johnson, "Application of a fuzzy object search technique to geophysical data processing," Nonlinear Image Processing, vol. 2180, pp. 300309, 1994. http://dx.doi.org/10.1117/12.172567

[31] L. Chen, H. D. Cheng, and J. Zhang, "Fuzzy subfiber and its application to seismic lithology classification," Information Science: Applications, vol. 1, no. 2, pp. 7795 , 1994. https://doi.org/10.1016/1069-0115(94)90009-4

[32] L. Chen, D. H. Cooley, and L. Zhang, "Intelligent data fitting technique for 3D velocity reconstruction," in Application and Science of Computational Intelligence, vol. 3390, pp. 103112, 1998. https://doi.org/10.1117/12.304794

[33] L. Chen and O. Adjei, "-connected segmentation and fitting," in Proceedings of IEEE Conference on System, Man and Cybernetics, Hague, The Netherlands, 2004, pp. 35003506. https://doi.org/10.1109/icsmc.2004.1400884

[34] L. Chen, "The necessary and sufficient condition and the efficient algorithms for gradually varied fill," Chinese Science Bulletin, vol. 35, no. 10, pp. 870-873, 1990. 
[35] L. Chen, Digital Functions and Data Reconstruction: Digital-Discrete Methods. New York: Springer, 2013.

[36] L. Chen, "Lambda-connectedness determination for image segmentation," in Proceedings of 36th IEEE Applied Image Pattern Recognition Workshop, Washington, DC, 207, pp. 7179. https://doi.org/10.1109/AIPR.2007.8

[37] J. N. Kapur, P. K. Sahoo, and A. K. C. Wong, "A new method of gray level picture thresholding using the entropy of the histogram," Computer Vision, Graphics, and Image Processing, vol. 29, no. 3, pp. 273285, 1985. https://doi.org/10.1016/0734-189x(85)90125-2

[38] V. B. S. Prasath, "Color image segmentation based on vectorial multiscale diffusion with inter-scale linking," in Pattern Recognition and Machine Intelligence, vol. 5909, pp. 339344, 2009. https://doi.org/10.1007/978-3-642-11164$8 \_55$

[39] P. Meer, C. A. Sher, and A. Rosenfeld, "The chain pyramid: Hierarchical contour processing," IEEE Transactions on Pattern Analysis and Machine Intelligence, vol. 12, no. 4, pp. 363376, 1990. https://doi.org/10.1109/34.50622

[40] C. Mathieu, I. E. Magnin, and C. Baldy-Porcher, "Optimal stochastic pyramid: segmentation of MRI data," in Medical Imaging VI: Image Processing, vol. 1652, pp. 1422, 1995. http://dx.doi.org/10.1117/12.59407

[41] S. C. Bansal, N. Khandelwal, D. V. Rai, R. Sen, S. K. Bhadada, K. A. Sharma, and N. Goswami, "Comparison between the QCT and the DEXA scanners in the evaluation of BMD in the lumbar spine," Journal of Clinical and Diagnostic Research, vol. 5, no. 4, pp. 694699, 2011.

[42] D. Mikesell, and I. V. Hicks, "Image segmentation via weighted carving decompositions," in Combinatorial Image Analysis, vol. 10256, pp. 268-279, 2017. https://doi. org/10.1007/978-3-319-59108-7_21

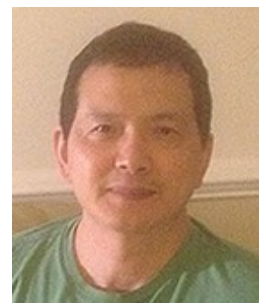

Li Chen is currently an Associate Professor of computer science at the University of the District of Columbia. He received his BS, MS, and $\mathrm{PhD}$ in CS from Wuhan University (1982), Utah State University (1995), and the University of Bedfordshire (2001), respectively. Chen's research interests are broad in computer science and applied mathematics and include applied algorithm design, digital and discrete geometry, image processing, and applications to data science. He has made contributions to several research areas of computer science and its applications including : (1) lambda-connected image segmentation methods, (2) fuzzy relation equations, (3) the digital form of the Gauss-Bonnet theorem, (4) the polynomial time algorithm for finite Abelian group decomposition, (5) the definition of digital manifolds and classification of 3D digital surfaces, and (6) the optimum algorithm for the check matrix of the optimal SEC-DED code (optimal Hamming code). In 2014, Chen chaired the Satellite Conference on Data Science of International Congress of Mathematicians (ICM14). He is an ACM Distinguished Speaker since 2015.

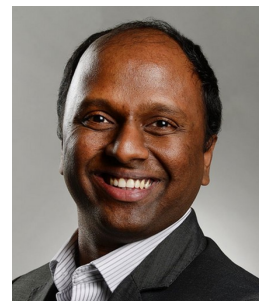

Surya Prasath is an assistant professor (research) in the Computer Science Department at the University of Missouri, USA. He received his PhD in Mathematics from the Indian Institute of Technology Madras, India in 2009 (defended in March 2010). He has been a postdoctoral fellow at the Department of Mathematics, University of Coimbra, Portugal, for two years. Since 2012 he is with the Computational Imaging and VisAnalysis (CiVA) Lab at the University of Missouri, USA working on various mathematical image processing and computer vision problems. He had summer fellowships/visits at Kitware Inc. NY, USA, The Fields Institute, Canada, and IPAM, University of California Los Angeles (UCLA), USA. His main research interests include nonlinear PDEs, regularization methods, inverse \& ill-posed problems, variational, PDE based image processing, and computer vision with applications in remote sensing, medical imaging domains. 